\title{
INVESTIGATION OF PLANAR AND CONFORMAL PRINTED ARRAYS FOR MIMO PERFORMANCE ANALYSIS
}

\author{
Celal Alp Tunc, Erdinc Irci, Onur Bakir, Defne Aktas, Vakur B. Erturk, and Ayhan Altintas \\ Dept. of Electrical and Electronics Engineering, Bilkent University, Ankara, Turkey, e-mail: celal@ee.bilkent.edu.tr
}

\begin{abstract}
MIMO channel capacity of printed arrays with dipole elements is analyzed. A MIMO channel model based on electric fields is used. The effects of mutual interactions among the array elements through space and surface waves are included into the channel matrix using a fullwave hybrid Method of Moments (MoM)/Green's function technique in the spatial domain. MIMO capacity of printed arrays is then compared with that of free standing thin wire dipole arrays. Results show better performance of printed arrays.
\end{abstract}

Key words: MIMO; planar printed arrays; conformal printed arrays; mutual coupling.

\section{INTRODUCTION}

Multiple Input Multiple Output (MIMO) wireless communication systems have been a focus of interest, due to their ability to increase the capacity in rich scattering environments by using multi-element antenna arrays both at the transmitter and the receiver side $[1,2]$.

One of the fundamental issues concerning the MIMO systems is the choice of the array type and configuration. Although printed arrays are advantageous over other antenna types for their low cost, light weight and conformability to the mounting surface, their performance in MIMO applications are not investigated adequately.

Dealing with multi-element antenna arrays, mutual coupling becomes significant. Effects of mutual coupling were successfully included into the MIMO channel matrix in [3] for uniform linear arrays of free standing thin wire dipoles (FSLA). Using the same idea, [4] investigated the MIMO performance of FSLA in detail; and [5] has recently expanded the work for planar and cylindrical arrays of free standing dipoles.

Mutual coupling effects were included into the channel model for FSLA in [3]-[5] by using the coupling matrices, obtained from the mutual interactions matrix. Ignoring the mutual coupling effects, these coupling matrices become the identity matrix. This method is appropriate when dealing with only one kind of array. However, if the comparison of various array types is desired, the method fails, since, when mutual coupling is ignored, all types give exactly the same channel capacity, when the channel models given in [3]-[5] are used.

Instead, a model with electric fields, which is described in Section 2, is used in this paper; and the MIMO channel capacity of printed linear dipole arrays is analyzed. The effects of mutual interactions among the array elements through space and surface waves are included into the channel matrix using a full-wave hybrid Method of Moments (MoM)/Green's function technique in the spatial domain. Two different Green's function representations are used. Basically, the efficient integral representation of the planar microstrip dyadic Green's function is used around the source region (diagonal and nearly diagonal terms of the impedance matrix), and highfrequency based asymptotic closed-form representation of the grounded dielectric slab Green's function is used when the field point is away from the source region [6].

MIMO capacity of printed arrays is then compared with that of FSLA. The geometric parameters of FSLA and printed arrays are chosen to be the same. Numerical results demonstrate the superiority of printed arrays over FSLA. More results on the MIMO performance of planar and conformal printed arrays will be presented during the presentation.

Organization of the paper is as follows: The MIMO system model is discussed in Section 2, then mutual coupling is addressed. Section 4 presents the numerical results. Finally, concluding remarks are given. An $e^{j w t}$ time convention is used and suppressed from the expressions.

\section{MIMO SYSTEM MODEL}

The scattering environment is a two dimensional (2D), single-bounce geometric model adopted from [3] and shown in Fig 1. It assumes a receiver and a transmitter array with a local cluster of scatterers around the transmitter. The local cluster is a disk of radius $R_{D}$ and it includes $S$ uniformly distributed scatterers. 


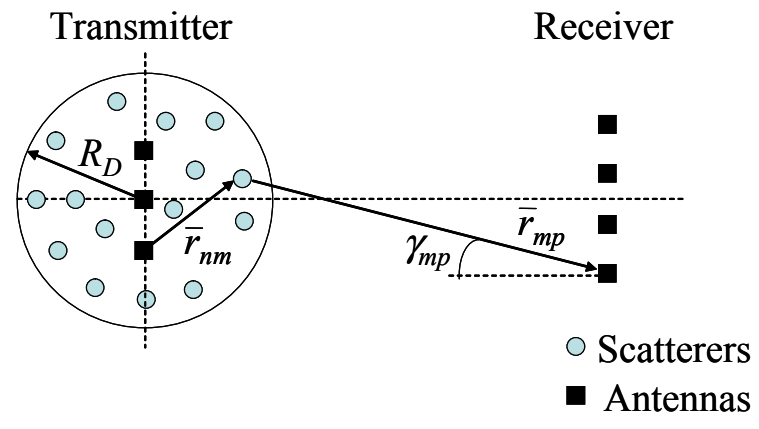

Figure 1. Scatterer Scenario.

Assuming flat fading, the received signal, $\bar{v}^{r x}$, can be written in terms of the transmitted one, $\bar{v}^{t x}$, and the additive white Gaussian noise with unit variance, $\bar{n}$, as:

$$
\bar{v}^{r x}=\mathbf{H} \bar{v}^{t x}+\bar{n} .
$$

In Eq. $1, \mathbf{H}$ denotes the $R \times T$ channel matrix, where $R$ and $T$ are the number of antenna elements in receiver and transmitter arrays, respectively. Assuming channel knowledge only at the receiver, the Shannon capacity, that is the maximum amount of data to be transmitted reliably, can be evaluated as

$$
\mathcal{C}=\log _{2}\left(\left|\mathbf{I}+\frac{P_{T}}{T} \mathbf{H H}^{*}\right|\right)
$$

where $I$ is the $R \times R$ identity matrix, $|$.$| is the matrix$ determinant, $P_{T}=E\left[\bar{v}^{t x *} \bar{v}^{t x}\right]$ is the total transmitted power, ${ }^{*}$ and $E[$.$] denotes the conjugate transpose and$ expectation operations, respectively.

The channel model in [3] is based on the phase differences due to scatterers and spatial properties of antenna elements. Here, it is modified in order to involve the electric fields, by splitting the channel matrix $\mathbf{H}$ into two as $\mathbf{H}_{1}$ and $\mathbf{H}_{2} . \mathbf{H}_{1}$ is the $S \times T$ transmission link matrix relating the transmitter (TX) to scatterers; whereas $\mathbf{H}_{2}$ $(R \times S)$ is the one linking scatterers to the receiver $(\mathrm{RX})$. Reader may refer to [7] for a similar model.

The overall channel matrix, $\mathbf{H}$ can then be expressed as:

$$
\mathbf{H}=\mathbf{H}_{2} \mathbf{H}_{1},
$$

and hence, Eq. 1 becomes

$$
\bar{v}^{r x}=\mathbf{H}_{2} \mathbf{H}_{1} \bar{v}^{t x}+\bar{n} .
$$

Using the electric fields, the $(m, n)$ th entry of $\mathbf{H}_{1}$ is written as:

$$
h_{m, n}^{(1)}=E^{t x}\left(\bar{r}_{n m}\right), \quad v_{n}^{t x}=1 \mathrm{~V}, \quad v_{i \neq n}^{t x}=0
$$

where $\bar{r}_{n m}$ is the position vector between the $n$th TX element and $m$ th scatterer. $E^{t x}\left(\bar{r}_{n m}\right)$ denotes the electric field generated by transmitter array, impinging on the $m$ th scatterer, when $n$th TX antenna is activated. The incident energy is scattered at the scatterers. Then, treating each scatterer as an isotropic radiator, the $(p, m)$ th entry of of $\mathbf{H}_{2}$ is expressed as follows

$$
h_{p, m}^{(2)}=\alpha_{m} \frac{e^{-j k r_{p m}}}{r_{p m}} f_{p}^{r x}\left(\gamma_{p m}\right)
$$

where $\alpha_{m}$ is the normal distributed random variable, with zero mean and unit variance, denoting the scattering coefficient of $m$ th scatterer, $\exp \left(-j k r_{p m}\right) / r_{p m}$ is the propagation term for the isotropic radiator, $r_{p m}$ is the distance between the $m$ th scatterer and the $p$ th RX element, and finally, $f_{p}^{r x}\left(\phi_{p m}\right)$ defines the reception ability of the receiver, that is the value of the complex electric field pattern of the RX array in the $m$ th scatterer's direction, when the $p$ th antenna is active. $f_{p}^{r x}\left(\phi_{p m}\right) \exp \left(-j k r_{p m}\right) / r_{p m}$ term is in fact nothing but the electric field of the RX on the $m$ th scatterer, when $p$ th element is active (i.e. $\left.E^{r x}\left(\bar{r}_{p m}\right)\right)$. Therefore, Eq. 6 becomes,

$$
h_{p, m}^{(2)}=\alpha_{m} E^{r x}\left(\bar{r}_{p m}\right), \quad v_{p}^{r x}=1 \mathrm{~V}, \quad v_{q \neq p}^{r x}=0
$$

for $p, q=1,2, \ldots, R$. It is noted that, the reciprocity principle also implies the result in Eq. 7.

\section{MUTUAL COUPLING}

The near field generated by an activated element in an array interacts with the neighboring elements and induces a current on them, causing all elements to radiate. These interactions are called mutual coupling. Mutual impedances are used to model the mutual coupling effects by relating the activation voltages, $\bar{V}$, with the induced currents, $\bar{I}$ as

$$
\bar{V}=\mathbf{Z} \bar{I}
$$

where $\mathbf{Z}$ is the mutual interactions matrix; whose diagonal elements $\left(z_{n n}\right)$ are the self impedances of array elements, and off-diagonal entries $\left(z_{n m}\right)$ represent the mutual impedances between them. Rewriting Eq. 8 explicitly, entries of the mutual impedance matrix can be expressed by,

$$
z_{n m}=\frac{v_{n}}{i_{m}}, \quad i_{j \neq m}=0
$$

for $j, m, n=1,2, \ldots, N$, where $N$ is the total number of elements in the array, $v_{n}$ is the activation voltage on the $n$th antenna, and $i_{m}$ is the induced current on the $m$ th element.

\subsection{Free Standing Linear Dipole Array (FSLA)}

In order to generate the mutual interactions matrix for FSLA, the method in [8] is used. For $z$-directed, sideby-side free standing arrays of thin wire dipoles, mutual impedances are evaluated by

$$
z_{n m}=-\frac{1}{i_{m}} \frac{1}{i_{n}} \int_{-h_{n}}^{h_{n}} E_{n m}(z) i_{n}(z) d z
$$




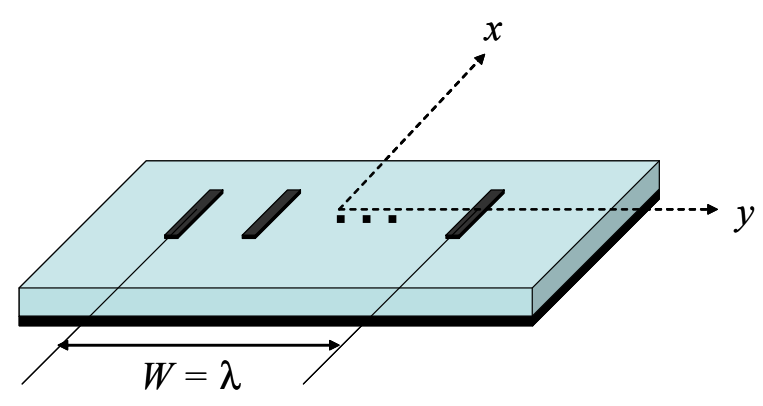

Figure 2. Fixed size PLA geometry.

incident on antenna- $n$, and $h_{n}$ is the half-length of the $n$th dipole. Assuming piecewise sinusoidal currents on the elements such as $i_{m}(z)=i_{m} \sin \left[k\left(h_{m}-|z|\right)\right] / \sin k h_{m}$, Eq. 10 becomes

$$
\begin{aligned}
z_{n m}= & \frac{j}{4 \pi \sin k h_{m} \sin k h_{n}} \int_{-h_{n}}^{h_{n}} F(z) d z \\
F(z)= & {\left[\frac{e^{-j k R_{1}}}{R_{1}}+\frac{e^{-j k R_{2}}}{R_{2}}-2 \cos k h_{m} \frac{e^{-j k R_{0}}}{R_{0}}\right] } \\
& \cdot \sin \left[k\left(h_{n}-|z|\right)\right]
\end{aligned}
$$

where $k$ is the free space wave number, and $\eta$ denotes the wave impedance. $R_{0}, R_{1}$ and $R_{2}$ are the distances from the center, top and bottom of the $n$th antenna, respectively. Note that, reciprocity implies $z_{m n}=z_{n m}$.

For the calculation of self impedances $\left(z_{n n}\right), R_{0}, R_{1}$ and $R_{2}$ are taken as: $R_{0}=\sqrt{a_{n}^{2}+z^{2}}, R_{1}=$ $\sqrt{a_{n}^{2}+\left(z-h_{n}\right)^{2}}$ and $R_{2}=\sqrt{a_{n}^{2}+\left(z+h_{n}\right)^{2}}$, where $a_{n}$ is the radius of the $n$th thin wire dipole element.

\subsection{Printed Linear Array (PLA)}

The investigation of one dimensional uniform linear array of printed dipoles is done utilizing the more general two dimensional finite array of printed dipoles [9]. An electric field integral equation (EFIE) is formed by enforcing the boundary condition that the total $E_{u}$ field must vanish on the dipole surfaces, where $\hat{u}$ is the direction of dipoles. A hybrid method based on the combination of method of moments (MoM) with the spatial domain Green's function is used to solve the aforementioned EFIE. The electric surface current density on each dipole is expanded in terms of one piecewise sinusoidal mode, which is found to be successful in [9] and [10]. Assuming an ideal delta gap generator at the terminals of each center-fed dipole and using Galerkin's MoM solution, the following matrix equation is obtained:

$$
\left[\mathbf{Z}+\mathbf{Z}_{T}\right] \bar{I}=\bar{V}
$$

where $\mathbf{Z}_{T}$ is the diagonal generator terminating impedance matrix. For the entries of the mutual interactions matrix $\mathbf{Z}$, reader may refer to $[6,9,10]$.

\begin{tabular}{|c|c|c|}
\hline & $z_{11}(\Omega)$ & $z_{12}(\Omega)$ \\
\hline FSLA & $73.1+j 40.7$ & $-12.5-j 29.9$ \\
\hline PLA & $43.5+j 447.1$ & $-3.1+j 2.3$ \\
\hline
\end{tabular}

Table 1. Mutual interactions matrix

Table 1 shows the self and mutual impedance values both for a PLA and a FSLA. Arrays are formed of two $\lambda / 2$ height dipoles, located side-by-side with a $\lambda / 2$ spacing. Elements of PLA are $\lambda / 100$ width, and placed on top of a dielectric substrate with a dielectric constant of $\epsilon_{r}=3.25$ and a thickness of $d=0.06 \lambda$ above a ground plane. The radius of the elements of FSLA is $\lambda / 200$.

\subsection{MIMO Channel Matrix with Mutual Coupling}

Mutual coupling effects were included into the channel model for FSLA in [3]-[5] using the coupling matrix, $\mathbf{C}$, obtained from mutual impedances matrix, $\mathbf{Z}$ as follows:

$$
\begin{gathered}
\mathbf{C}_{R}=\left(Z_{A}+Z_{T}\right)\left(\mathbf{Z}+Z_{T} \mathbf{I}\right)^{-1} \\
\mathbf{H}^{\prime}=\mathbf{C}_{R} \mathbf{H}
\end{gathered}
$$

where $\mathbf{C}_{R}(R \times R)$ is the coupling matrix for the receiver. $Z_{A}$ is the antenna impedance and $Z_{T}$ represents load impedances at the receiver. In order to have a conjugate match, $Z_{T}=Z_{A}^{*}$ can be chosen. Finally, $\mathbf{H}^{\prime}$ stands for the mutual coupling effect included channel matrix. Ignoring the mutual coupling, $\mathbf{C}_{R}$ becomes the $R \times R$ identity matrix and $\mathbf{H}^{\prime}=\mathbf{H}$, because of the normalization with $Z_{A}+Z_{T}$. This is a brilliant method, when dealing with only one kind of array (i.e. FSLA or PLA). However, comparing different array types, such as PLA versus FSLA, the method fails, since no mutual coupling cases for both types give exactly the same channel matrix, using the channel models in [3]-[5].

Instead, the model with electric fields, which is described in Section 2, is used in this paper with the following procedure for the entries of $\mathbf{H}_{1}$ :

i. Evaluate mutual interactions matrix of the transmitter, $\mathbf{Z}_{t x}$.

ii. Start with $n=1$.

iii. Activate $n$th TX element, such that $v_{n}^{t x}=$ $1 \mathrm{~V}, v_{j \neq n}^{t x}=0$.

iv. Calculate the current vector for $\mathrm{TX}, \bar{i}^{t x}=\mathbf{Z}_{t x}^{-1} \bar{v}^{t x}$.

v. Find

$$
h_{m, n}^{(1)}=E^{t x}\left(\bar{r}_{n m}\right)=\sum_{t=1}^{T} E\left(i_{t}, m\right)
$$

where $E\left(i_{t}, m\right)$ is the electric field due to the current $i_{t}$ on the $t$ th element in the transmitter array, impinged on $m$ th scatterer, for $j, n, t=1,2, \ldots, T$. 


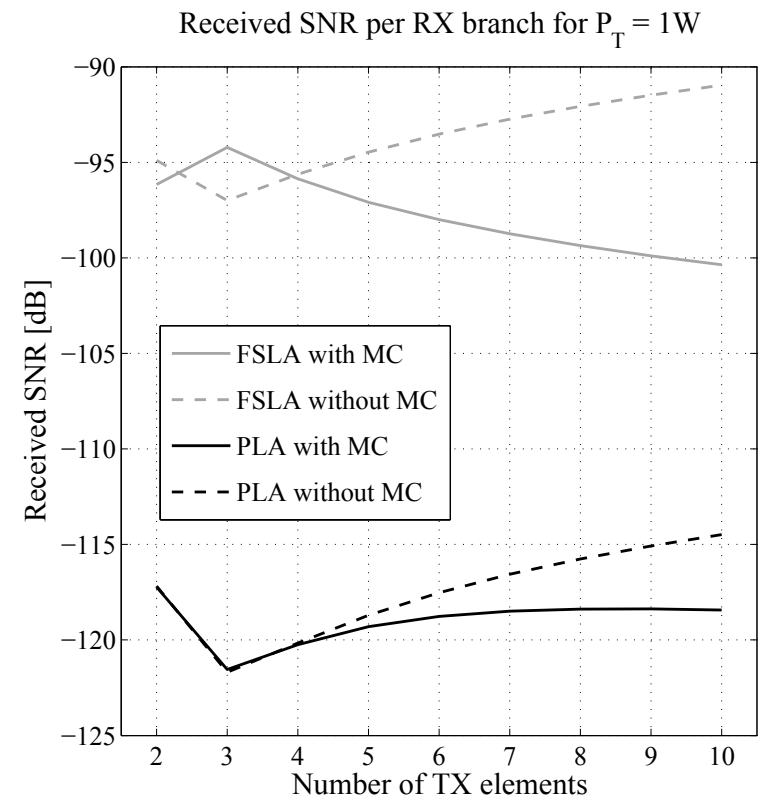

Figure 3. Received SNR for $P_{T}=1 W$.

vi. Increase $n$, and go to (iii).

For FSLA, the electric field of the thin wire dipole, incident on the $m$ th scatterer, located on its azimuthal plane, is [11],

$$
E\left(i_{t}, m\right)=j \eta \frac{i_{t} e^{-j k r_{t m}}}{2 \pi r_{t m} \sin k h_{t}}\left[1-\cos k h_{t}\right]
$$

where $r_{t m}$ is the distance between the $t$ th transmit antenna and $m$ th scatterer. For PLA, reader may refer to [9] for the electric field of a single printed element.

Forced excitation case $\left(Z_{T}=0\right)$ is considered in this work. However, the method is capable of the free excitation case $\left(Z_{T} \neq 0\right)$ as well, by simply calculating the current vector by $\bar{i}^{t x}=\left[\mathbf{Z}_{t x}+\mathbf{Z}_{T}\right]^{-1} \bar{v}^{t x}$, where $\mathbf{Z}_{T}$ is the diagonal matrix whose entries are the termination impedances of each antenna element.

\section{NUMERICAL RESULTS}

MIMO performance of printed linear arrays of dipole elements are investigated in terms of mean channel capacity. The channel is modeled by locating $S=100$ uniformly distributed scatterers around the transmitter within a disk of radius of $R_{D}=200 \lambda$, on the plane perpendicular to the current direction of the antenna elements. The receiver array is assumed to be a free standing linear array (FSLA) located $2000 \lambda$ away from the transmitter in a broadside manner, formed by $R=10$ uniform linear dipoles, where each of them is separated by a distance of $\lambda / 2$. Mean capacity results are obtained by averaging the MIMO channel capacity over 500 channel realizations.
Printed vs Free Standing Linear Array for fixed size

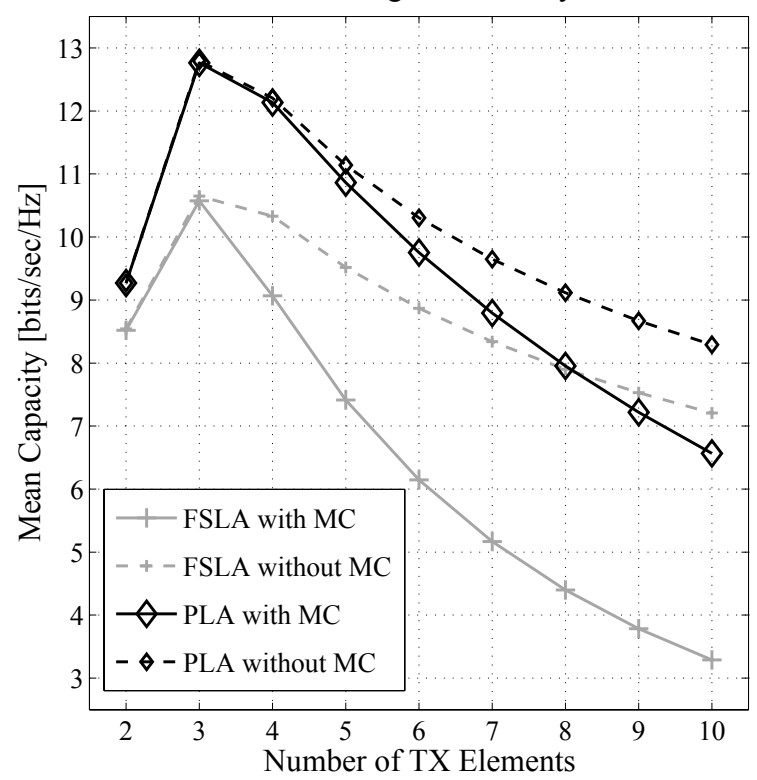

Figure 4. Mean capacity for fixed size TX array.

PLA analyzed here are formed by uniform dipoles of $\lambda / 2$ height and $\lambda / 100$ width, which are placed on top of a dielectric substrate with a dielectric constant of $\epsilon_{r}=3.25$ and a thickness of $d=0.06 \lambda$ above a ground plane. FSLA are considered to be composed of thin wire dipole elements of $\lambda / 2$ height and $\lambda / 200$ radius.

\subsection{Fixed Size Arrays}

Fixed size PLA at the transmitter is considered. The width of the PLA is limited to $W=\lambda$ as illustrated in Fig. 2, and the number of transmitter elements is increased from 2 to 10 so as to have equally spaced antenna elements. Results are compared with FSLA of the same geometric parameters. Fig. 3 shows the results for the received signal to noise ratio (SNR) per receiver array element, averaged over 500 channel realizations, both with and without mutual coupling, when the total transmitted power is taken as $P_{T}=1 \mathrm{~W}$, which is allocated equally to each antenna element of TX array. Cases without mutual coupling are obtained by taking off-diagonal entries of mutual impedance matrices as zero. Results clearly show that, for a fixed transmit power, received power in FSLA case is drastically higher. Furthermore, increasing the number of elements for a limited array size, the received SNR decreases due to mutual coupling effects. Inspecting the FSLA case, it is noted that, around $\lambda / 2$ interelement spacing, mutual coupling has a constructive effect on the received SNR. This effect is due to the distorted radiation pattern $[3,4,12]$.

MIMO capacities are calculated adjusting the transmitted power $\left(P_{T}\right)$ to have $10 \mathrm{~dB}$ received SNR in no mutual coupling cases both for PLA and FSLA. It is noted 


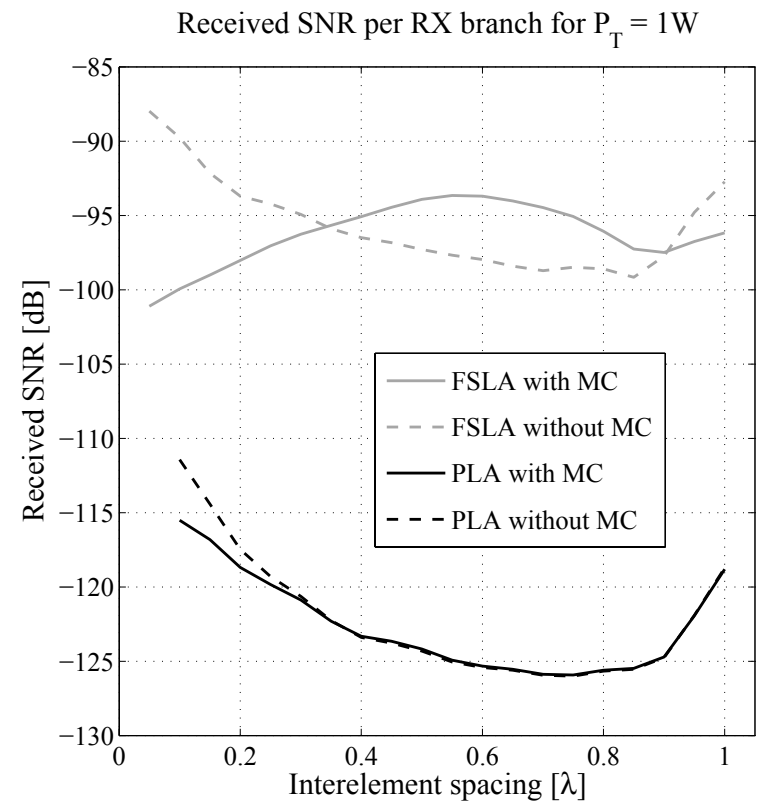

Figure 5. Received SNR for $P_{T}=1 \mathrm{~W}$.

that, for a desired received SNR, PLA must be fed with a power approximately $25 \mathrm{~dB}$ more than that of an FSLA. Mean capacity results are plotted versus increasing number of TX elements in Fig. 4. Increasing the number of elements for a fixed array width, thus decreasing the interelement spacing, capacity is reduced due to the signal correlation induced by the channel beyond 3 elements. Mutual coupling further decreases the capacity. In particular, ignoring mutual coupling in FSLA results in significant overestimation of the capacity for small interelement spacings.

For a fixed received SNR, PLA have higher MIMO channel capacity than FSLA. Moreover, it suffers less from the mutual coupling, since the mutual to self impedance ratio is smaller compared to FSLA (Table 1). Optimum number of antenna elements for this geometry appears to be 3 for both cases, which yields a $\lambda / 2$ interelement spacing. Also, for $\lambda / 2$ spacing, mutual coupling effects seem almost negligible.

\subsection{Arrays with Fixed Number of Elements}

PLA and FSLA with 6 elements are analyzed. Antenna element spacing is varied from $0.05 \lambda$ to $\lambda$. Plotting the received SNR for $P_{T}=1 \mathrm{~W}$ in Fig. 5, required transmitted powers for $10 \mathrm{~dB}$ SNR at RX are obtained for both PLA and FSLA without mutual coupling. Fig. 6 illustrates the mean capacity results for 6 element PLA and FSLA both with and without coupling. For a certain desired received SNR, PLA has a better MIMO performance than FSLA. Furthermore, mutual coupling affects FSLA more. Mutual coupling effects on the capacity become more significant for small interelement spacings, as expected. Optimum interelement spacing seems to be

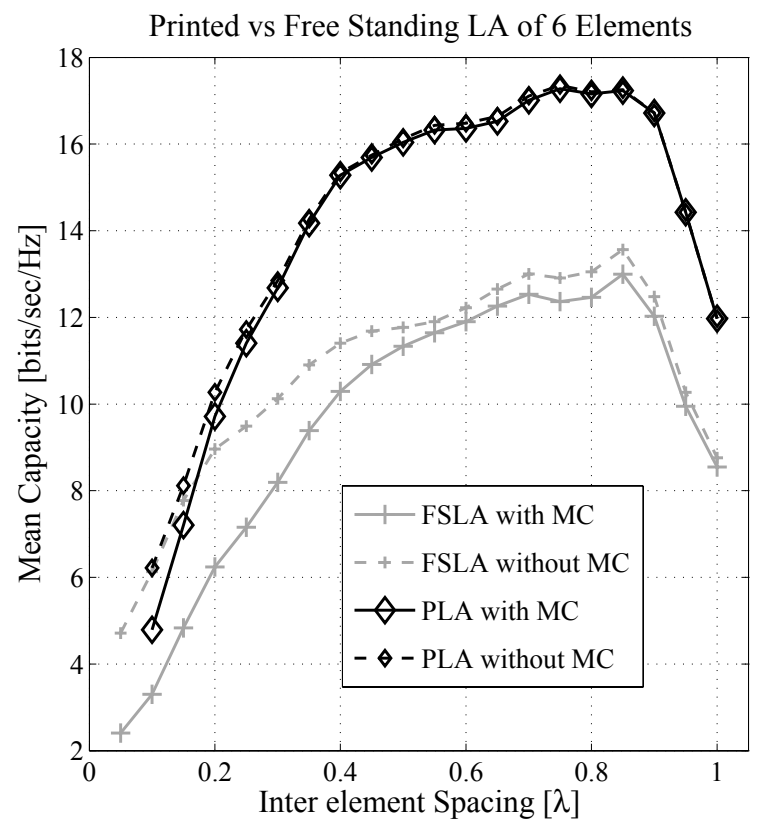

Figure 6. Mean capacity for 6 element TX array.

$0.75 \lambda$ for PLA, and $0.85 \lambda$ for FSLA. Increasing element spacing further, decreases the capacity due to the grating lobes in the radiation pattern.

\section{CONCLUSION}

MIMO performance of printed linear arrays (PLA) of dipole elements are investigated, both with and without mutual coupling, in terms of mean channel capacity. Comparisons with free standing thin wire dipole arrays (FSLA) are given. A conventional single bounce, 2D geometric channel model with local clustering is modified to involve electric field values and used for obtaining MIMO channel matrices.

As expected, mutual coupling effects on the capacity become more significant for small interelement spacings. PLA shows higher MIMO capacity than FSLA for a desired received SNR, though it must be fed with a power significantly higher than that for FSLA. Moreover, it suffers less from the mutual coupling, since the mutual to self impedance ratio is smaller compared to FSLA. Optimum interelement spacings are presented, and it is shown that, increasing interelement spacing beyond the optimum value decreases the capacity due to the grating lobes in the radiation pattern.

\section{ACKNOWLEDGMENTS}

This work has been supported in part by the European Union Network of Excellence (NoE) NEWCOM, under the 6th Framework Programme. 


\section{REFERENCES}

[1] Telatar I. E., "Capacity of multi-antenna Gaussian channels," Europ. Trans. Telecommun., vol. 10, pp. 585-595, Nov. 1999.

[2] Foschini, G. J. and Gans M. J., "On limits of wireless communications in a fading environment when using multiple antennas", Wireless Personal Commun., vol. 6, pp. 311-335, Mar. 1998.

[3] Svantesson T. and Ranheim A., "Mutual coupling effects on the capacity of multielement antenna systems," in Proc. IEEE Int. Conf. Acoustics, Speech, and Signal Processing, vol. 4, Salt Lake City, UT, May 711, 2001, pp. 2485-2488.

[4] Janaswamy R., "Effect of element mutual coupling on the capacity of fixed length linear arrays," IEEE Antennas Wireless Propagat. Lett., vol. 1, no. 1, pp. 157-160, 2002.

[5] Tunc C. A., Aktas D., and Altintas A., "On Arrays of Dipole Antennas with Mutual Coupling in MIMO Wireless Channels," 15th IST Mobile and Wireless Communications Summit, Mykonos, Greece, Jun. 48, 2006

[6] Marin M., Barkeshli S., and Pathak P. H., "Efficient analysis of planar microstrip geometries using a closed-form asymptotic representation of the grounded dielectric slab Green's function," IEEE Trans. Microwave Theory Tech., vol. 37, pp. 669-679, Apr. 1989.

[7] Getu B. N. and Andersen J. B., "The MIMO cube: A compact MIMO antenna," IEEE Trans. Wireless Commun., vol. 4, no. 3, pp. 1136-1141, May 2005.

[8] Elliott R. S., Antenna Theory \& Design, Revised Edition, Wiley IEEE Press, 2003

[9] Pozar D. M., "Analysis of finite phased arrays of printed dipoles," IEEE Trans. Antennas Propagat., vol. AP-33, pp. 1045-1053, Oct. 1985.

[10] Pozar D. M., "Input impedance and mutual coupling of rectangular microstrip antennas," IEEE Trans. Antennas Propagat., vol. AP-30, pp. 1191-1196, Nov. 1982.

[11] Balanis C. A., Antenna Theory: Analysis and Design, New York: Wiley, 1997.

[12] Ozdemir M. K., Arvas E., and Arslan H., "Dynamics of spatial correlation and implications on MIMO systems," IEEE Commun. Mag., vol. 42, no. 6, pp. 514-519, Jun. 2004. 